\title{
Optical polarization of neutron-rich sodium isotopes and $\beta$-NMR measurements of quadrupole moments
}

\author{
M. Keim $\dagger$, U. Georg $\dagger$, A. Klein $\dagger$, R. Neugart $\dagger$, M. Neuroth $\dagger$, S. Wilbert $\dagger$, \\ P. Lievens $\frac{\ddagger}{\ddagger}$ L.Vermeeren $₫$ and the ISOLDE Collaboration $\S$ \\ $\nmid$ Institut für Physik, Universität Mainz, D-55099 Mainz \\ Laboratorium voor Vaste-Stoffysika en Magnetisme, K.U. Leuven, \\ B-3001 Leuven, Belgium \\ I Instituut voor Kern- en Stralingsfysika, K.U. Leuven, B-3001 Leuven, Belgium \\ $\S$ CERN, CH-1211 Geneva 23, Switzerland
}

\begin{abstract}
The nuclear quadrupole moments of neutron-rich sodium isotopes are being investigated with the help of in-beam polarization by optical pumping in combination with $\beta$-NMR techniques. First measurements have yielded the quadrupole splittings of NMR signals in the lattice of $\mathrm{LiNbO}_{3}$ for the isotopes ${ }^{26} \mathrm{Na},{ }^{27} \mathrm{Na}$ and ${ }^{28} \mathrm{Na}$. Interaction constants and ratios of the electric quadrupole moments are derived. In view of future experiments, $\beta$-decay asymmetries for the sequence of isotopes up to the $N=20$ neutron shell closure, ${ }^{26-31} \mathrm{Na}$, have been measured.
\end{abstract}

(IS 304)

Contribution to the 10th International

Conference on Hyperfine Interactions,

Leuven, 28 August - 1 September 1995

(To be published in Hyperfine Interactions) 


\section{Introduction}

Studies of the properties of very neutron-rich sodium and magnesium isotopes around the neutron number $N=20$ have revealed peculiar features. A series of experimental and theoretical investigations has been initiated by the direct mass measurements on sodium isotopes $[1,2]$. Binding energies much larger than predicted by pure sd-shell calculations [3] were observed for ${ }^{31} \mathrm{Na}$ and ${ }^{32} \mathrm{Na}$. This and the subsequent observation of a remarkably low-lying level in ${ }^{32} \mathrm{Mg}$, assigned to a $2^{+}$state [4], have been interpreted as an evidence of deformation. The recently measured large $\mathrm{B}(\mathrm{E} 2)$ value [5] confirms this conclusion. Laser spectroscopy measurements of the changes in mean square charge radii for the sodium isotopes up to ${ }^{31} \mathrm{Na}[6]$ indicate a considerable increase of $\left\langle r^{2}\right\rangle$ at $A=29$ which is also in accordance with this interpretation.

Theoretically, the nuclear deformation at $N \simeq 20, Z \simeq 10-12$, increasing the binding, is caused by $\mathrm{np}-\mathrm{nh}$ neutron excitations to the fp-shell. This has been shown from Hartree-Fock calculations [7] as well as from shell-model calculations using an extended model space $[8,9]$. Compared to former calculations restricted to the sd-shell and describing nuclei closer to stability quite well [3], the latter could improve the agreement with the experimental binding energies significantly.

A measurement of the electromagnetic moments is important for the understanding of nuclear structure, particularly in view of improvements in the predictive power of shell-model calculations. Precise values of the magnetic moments of neutron-rich sodium isotopes up to $N=20$ were obtained from hyperfine structure measurements performed by high-resolution laser spectroscopy [6]. Electric quadrupole moments are particularly sensitive to deformation. However, for light elements it is very difficult to resolve the small quadrupole interaction of the atomic hyperfine structure. That is why double resonance experiments by Touchard et al. [10] yielded only upper limits of the order 100 mbarn for the quadrupole moments of ${ }^{26-29} \mathrm{Na}$. 


\section{Experiment}

In the experiments described here, the technique of collinear fast beam laser spectroscopy is combined with $\beta$-emission detected nuclear magnetic resonance $(\beta$-NMR) [11]. The efficient optical excitation by laser light is used to polarize the nuclear spins. The small interaction of the nuclear quadrupole moment with an internal electric field gradient of a crystal lattice is accessible to a measurement due to the high resolution of NMR. A schematic picture of the experimental setup is shown in Figure 1.

The experiments are performed at the PS-Booster-ISOLDE facility of CERN. The radioactive sodium isotopes are produced by fission of uranium or thorium in a $\mathrm{UC}_{2}$ or $\mathrm{ThC}_{2}$ target which is irradiated with the $1 \mathrm{GeV}$ proton beam from the PSBooster. A radioactive beam of singly-charged ions is extracted from a surface ion source, electrostatically accelerated to $60 \mathrm{keV}$ and mass-separated. After neutralization in an alkali-metal vapour, the sodium atoms are transmitted through the optical excitation region. Here, in a weak longitudinal magnetic field, the atoms interact with the collinearly superimposed circularly polarized laser beam. The atoms are optically pumped in the D2-transition $3 \mathrm{~s}^{2} \mathrm{~S}_{1 / 2} \rightarrow 3 \mathrm{p}{ }^{2} \mathrm{P}_{3 / 2}$ at $589 \mathrm{~nm}$ from the $F=I+1 / 2$ hyperfine component of the ground state and thus become spin-polarized. The polarization of the valence electrons is transferred to the nuclei via the hyperfine interaction. In this process a nuclear polarization of $50-80 \%$ can be achieved, depending on the nuclear spin and the hyperfine splitting.

The spins of the polarized sodium isotopes are rotated adiabatically into the direction of the strong transverse magnetic field of the $\beta$-NMR setup. Here, the atoms are implanted into an appropriate host crystal lattice. As a consequence of the nuclear polarization, the angular distribution of the emitted $\beta$-particles is asymmetric with respect to the magnetic field axis. This is detected with the help of two scintillator telescopes placed at $0^{\circ}$ and $180^{\circ}$ with respect to the field direction. Radiofrequency (rf) is applied to a pair of coils irradiating the sample and inducing transitions between the nuclear Zeeman levels. At resonance the levels coupled by the rf field become equally populated, and the polarization and thus the decay 
asymmetry is reduced. The measuring sequence has to allow for the proton pulse structure (typically one pulse every $4.8 \mathrm{~s}$ ) which defines the natural stepping time for the radiofrequency. The integration time for each channel of about 3 half-lives starts with the impact of the protons on the production target. During this time the rf is continuously applied to the sample.

In a cubic host lattice the equidistant level spacing is given by the Larmor frequency which corresponds to the magnetic dipole moment and the nuclear spin $I$. For a measurement of the electric quadrupole moment, the nuclei are implanted into a host lattice with non-cubic symmetry. There, the charge distribution around certain lattice sites results in a non-vanishing electric field gradient $\left\langle\phi_{Z Z}\right\rangle$. The additional interaction with the electric quadrupole moments shifts the Zeeman levels and causes a splitting of the NMR signal into $2 \cdot I$ equidistant components. This splitting is given by the product of the nuclear quadrupole moment $Q_{S}$ and the field gradient $\left\langle\phi_{Z Z}\right\rangle$ at the lattice site of the implanted nuclei.

\section{Results}

The production yields of all isotopes up to ${ }^{31} \mathrm{Na}$ were found to be sufficient for the detection of $\beta$-decay asymmetries. These asymmetries vary between $18 \%$ and $40 \%$, and they are roughly consistent with the known $\beta$-decay properties and the assumption of $50 \%$ nuclear polarization. The detailed results are given in Table 1, together with the typical number of counts in our detectors (about 10\% solid angle) produced by one proton pulse.

In the $\beta$-NMR experiments, it turned out that the search for an appropriate noncubic host crystal is not trivial. No quadrupole splitting could be resolved for ${ }^{26} \mathrm{Na}$ and ${ }^{27} \mathrm{Na}$ in a commercially available $\mathrm{NaNO}_{3}$ crystal of which the electric field gradient at the site of sodium nuclei is known. The same is true for metallic single crystals of magnesium and beryllium which are common non-cubic target materials for $\beta$-NMR studies [11]. In all these lattices the field gradient is obviously too small to give a resolved splitting for the surprisingly small quadrupole moments. Only in $\mathrm{LiNbO}_{3}$, where the quadrupole interaction of stable as well as radioactive lithium 
isotopes had been studied extensively $[12,13]$, well resolved structures for ${ }^{26} \mathrm{Na},{ }^{27} \mathrm{Na}$ and ${ }^{28} \mathrm{Na}$ were observed. Figures 2 and 3 show the parts of the spectra obtained for ${ }^{26} \mathrm{Na},{ }^{27} \mathrm{Na}$ and ${ }^{28} \mathrm{Na}$ which correspond to transitions between Zeeman levels with $\mathrm{m}_{I} \geq 0$. As in the polarization process the population is accumulated to levels with positive $\mathrm{m}_{I}$ values, the amplitudes of these lines are enhanced.

The solid curve is the result of a least square fit using equidistantly separated gaussians. For ${ }^{26} \mathrm{Na}$, the resonance of atoms implanted at lattice sites with very small or vanishing field gradient is taken into account by an additional line at the Larmor frequency.

The resonances obtained for the different isotopes exhibit rather different line widths. About 15 to $20 \mathrm{kHz}$ (FWHM) are observed for ${ }^{26} \mathrm{Na}$ and ${ }^{27} \mathrm{Na}$ which have very small quadrupole splittings. The broadening of the ${ }^{28} \mathrm{Na}$ line to about $200 \mathrm{kHz}$ roughly scales with the much larger quadrupole interaction constant. This indicates that quadrupole interaction is responsible for the line broadening.

The nuclear polarization of the investigated $\mathrm{Na}$ isotopes implanted in $\mathrm{LiNbO}_{3}$ is well preserved during the half-lives ranging from $1 \mathrm{~s}$ down to $30 \mathrm{~ms}$. A relaxation curve, i.e. the time dependence of the decay asymmetry, for ${ }^{26} \mathrm{Na}\left(T_{1 / 2}=1.07 \mathrm{~s}\right)$ is shown in Figure 4. The quantitative description of this curve has to account for at least two exponential decay components corresponding to $T_{1}=0.67 \mathrm{~s}$ and $4.64 \mathrm{~s}$. As the half-life of ${ }^{28} \mathrm{Na}\left(T_{1 / 2}=30.5 \mathrm{~ms}\right)$ is too short for a relaxation measurement, it is difficult to investigate the influence of the quadrupole interaction on the relaxation process.

The relaxation in other non-cubic host crystals also exhibits a two-component behaviour with $T_{1}$ values between 100 and $300 \mathrm{~ms}$ for the fast component and between 1 and $10 \mathrm{~s}$ for the slow one. The relative strengths of these components are quite different. Only in the cubic $\mathrm{NaF}$ the slow component of $T_{1}=14$ s clearly predominates. The total asymmetry extrapolated to the time of implantation varies between $15 \%$ $\left(\mathrm{NaNO}_{3}\right)$ and $32 \%(\mathrm{NaF})$. As the nuclear polarization achieved in the optical pumping of free atoms is independent of the crystal, this suggests a very fast unresolved relaxation component or a crystal dependent destruction of polarization during the implantation. 
The field gradient for sodium implanted in $\mathrm{LiNbO}_{3}$ is so far unknown, which means that no absolute values for the quadrupole moments can be deduced. However, as the field gradient is isotope-independent, the ratios of quadrupole moments can be extracted. These are listed in Table 2, together with the quadrupole interaction constants $\nu_{Q}=Q_{S} \cdot\left\langle\phi_{Z Z}\right\rangle \cdot e / h$. For comparison, the results of recent shellmodel calculations by Brown [14] are included. The ratio $Q_{S}\left({ }^{27} \mathrm{Na}\right) / Q_{S}\left({ }^{26} \mathrm{Na}\right)$ is in good agreement with the theoretical value, whereas only the sign and the order of magnitude is reproduced for the ratio $Q_{S}\left({ }^{28} \mathrm{Na}\right) / Q_{S}\left({ }^{26} \mathrm{Na}\right)$.

A rough estimate of $\left\langle\phi_{Z Z}\right\rangle$ for implanted sodium nuclei can be derived from the well-known interaction of lithium in $\mathrm{LiNbO}_{3}[12,13]$. The assumption that in the implantation process the sodium atoms come to rest at the lattice site of the chemically equivalent lithium yields (from the systematics of the Sternheimer antishielding correction) the order of magnitude $\left|\nu_{Q} / Q_{S}\right| \approx 12 \mathrm{kHz} / \mathrm{mbarn}$, corresponding to $\left|Q_{S}\left({ }^{26} N a\right)\right| \approx 12$ mbarn. This is compatible with the estimate of an upper limit $\left|Q_{S}\left({ }^{26} \mathrm{Na}\right)\right|<25$ mbarn which is derived from the shape of the unresolved spectrum measured in $\mathrm{NaNO}_{3}$ and the value of $\left\langle\phi_{Z Z}\right\rangle$ at the substitutional Na lattice site reported in literature [15]. The theoretical value by Brown, $Q_{S}\left({ }^{26} \mathrm{Na}\right)=-11.0 \mathrm{mbarn}$, also supports these assumptions.

\section{Conclusion and outlook}

We present the status of an investigation of the nuclear quadrupole moments of neutron-rich sodium isotopes performed at the PS-Booster-ISOLDE facility. It has been shown that the experimental method combining optical pumping of a fast radioactive beam with $\beta$-NMR techniques is suitable for the determination of quadrupole moment ratios. First runs yielded resolved NMR-spectra for ${ }^{26} \mathrm{Na},{ }^{27} \mathrm{Na}$ and ${ }^{28} \mathrm{Na}$ in a $\mathrm{LiNbO}_{3}$ single crystal. For the measurement of absolute values of quadrupole moments, it will be necessary to find an appropriate crystal lattice with good relaxation properties and a known electric field gradient for implanted sodium atoms.

In the future it should be possible to extend the measurements up to ${ }^{31} \mathrm{Na}$ at the 
$N=20$ shell closure. The decay asymmetries of all these isotopes lie between $18 \%$ and $40 \%$. Since the count rates decrease dramatically for the more neutron-rich isotopes (see Table 1), it will be necessary to use more efficent $\beta$-NMR techniques. In former measurements of the quadrupole moments of lithium isotopes, several radiofrequencies were applied simultaniously [13]. Similarly, an experiment on ${ }^{8} \mathrm{~B}$ used a sequential irradiation of the sample with the different resonance frequencies that correspond to a given quadrupole moment [16]. Both procedures yield strongly enhanced resonance signals, because all nuclear Zeeman states become coupled.

This work has been supported by the German BMFT under contract numbers 06 MZ $501 \mathrm{I}$ and $06 \mathrm{MZ} 566 \mathrm{I}$ and by the Belgian NFWO and IIKW. 


\section{Bibliography}

[1] C. Thibault, R. Klapisch, C. Rigaud, A.M. Poskanzer, R. Prieels, L. Lessard and W. Reisdorf, Phys. Rev. C 12 (1975) 644

[2] N.A. Orr, W. Mittig, L.K. Fifield, M. Lewitowicz, E. Plagnol, Y. Schutz, Zhan Wen Long, L. Bianchi, A. Gillibert, A.V. Belozyorov, S.M. Lukyanov, Y.E. Penionzhkevich, A.C.C. Villari, A. Cunsolo, A. Foti, G. Audi, C. Stephan and L. Tassan-Got, Phys. Lett. B 258 (1991) 29

[3] B.H. Wildenthal and W. Chung, Phys. Rev. C 22 (1980) 2260

[4] D. Guillemaud-Mueller, C. Detraz, M. Langevin, F. Naulin, M. de Saint-Simon, C. Thibault, F. Touchard and M. Epherre, Nucl. Phys. A426 (1984) 37

[5] T. Motobayashi, Y. Ikeda, Y. Ando, K. Ieki, M. Inoue, N. Iwasa, T. Kikuchi, M. Kurokawa, S. Moriya, S. Ogawa, H. Murakami, S. Shimoura, Y. Yanagisawa, T. Nakamura, Y. Watanabe, M. Ishihara, T. Teranishi, H. Okuno and R.F. Casten, Phys. Lett. B 346 (1995) 9

[6] G. Huber, F. Touchard, S. Büttgenbach, C. Thibault, R. Klapisch, H.T. Duong, S. Liberman, J. Pinard, J.L. Vialle, P. Juncar and P. Jacquinot, Phys. Rev. C 18 (1978) 2342

[7] X. Campi, H. Flocard, A. K. Kerman and S. Koonin, Nucl. Phys. A251 (1975) 193

[8] A. Poves and J. Retamosa, Nucl. Phys. A571 (1994) 221

[9] E.K. Warburton, J.A. Becker and B.A. Brown, Phys. Rev. C 41 (1990) 1147 
[10] F. Touchard, J.M. Serre, S. Büttgenbach, P. Guimbal, R. Klapisch, M. de SaintSimon, C. Thibault, H.T. Duong, P. Juncar, S. Liberman, J. Pinard and J.L. Vialle, Phys. Rev. C 25 (1982) 2756

[11] H. Ackermann, P. Heitjans and H.-J. Stöckmann, Topics in Current Physics, Vol. 31, ed. J. Christiansen, Springer-Verlag, Berlin, Heidelberg, New York, Tokyo 1983, p. 291

[12] E. Arnold, J. Bonn, W. Neu, R. Neugart, E.W. Otten and ISOLDE Collaboration, Z. Phys. A 331 (1988) 295

[13] E. Arnold, J. Bonn, A. Klein, R. Neugart, M. Neuroth, E.W. Otten, P. Lievens, H. Reich, W. Widdra and ISOLDE Collaboration, Phys. Lett. B 281 (1992) 16

[14] B.A. Brown and B.H. Wildenthal, Ann. Rev. Nucl. Part. Sci. 38 (1988) 29; B.A. Brown, private communication 1995

[15] R.V. Pound, Phys. Rev. 79 (1950) 685

[16] T. Minamisono, T. Ohtsubo, I. Minami, S. Fukuda, A. Kitagawa, M. Fukuda, K. Matsuta, Y. Nojiri, S. Takeda, H. Sagawa and H. Kitagawa, Phys. Rev. Lett. 69 (1992) 2058 


\section{Figure captions:}

Figure 1: Schematic picture of the experimental setup

Figure 2: NMR-spectra of ${ }^{26} \mathrm{Na}$ and ${ }^{27} \mathrm{Na}$ in $\mathrm{LiNbO}_{3}$ (c-axis parallel to the magnetic field)

Figure 3: NMR-spectrum of ${ }^{28} \mathrm{Na}$ in $\mathrm{LiNbO}_{3}$ (c-axis perpendicular to the magnetic field). The origin of the frequency scale is set to the Larmor frequency.

Figure 4: Relaxation curve of ${ }^{26} \mathrm{Na}$ in $\mathrm{LiNbO}_{3}$. The polarized sodium nuclei are implanted in the first $320 \mathrm{~ms}$ (16 channels).

\section{Table captions:}

Table 1: Count rates and $\beta$-decay asymmetries for ${ }^{26-31} \mathrm{Na}$ implanted in the cubic crystal lattice of $\mathrm{NaF}$

Table 2: Quadrupole interaction constants $\nu_{Q}$ and electric quadrupole moments of ${ }^{27} \mathrm{Na}$ and ${ }^{28} \mathrm{Na}$ (preliminary) relative to ${ }^{26} \mathrm{Na}$ 
Table 1:

\begin{tabular}{|c|cc|}
\hline A & exp. asym. [\%] & counts per pulse \\
\hline 26 & 32 & $1 \cdot 10^{7}$ \\
27 & 30 & $1 \cdot 10^{6}$ \\
28 & 40 & $4 \cdot 10^{4}$ \\
29 & 21 & $1 \cdot 10^{4}$ \\
30 & 18 & $4 \cdot 10^{3}$ \\
31 & 22 & $2 \cdot 10^{2}$ \\
32 & $?$ & $<20$ \\
\hline
\end{tabular}

Table 2:

\begin{tabular}{|c|c|c|c|}
\hline \multirow{2}{*}{$\mathrm{A}$} & $\left|\nu_{Q}\right|$ & \multicolumn{2}{|c|}{$Q_{S} / Q_{S}\left({ }^{26} \mathrm{Na}\right)$} \\
& {$[\mathrm{kHz}]$} & exp. & theor. $[14]$ \\
\hline 26 & $142.6(3.3)$ & 1 & 1 \\
27 & $198.6(2.4)$ & $1.39(4)$ & 1.09 \\
28 & $1099(22)$ & $-7.7(2)$ & -3.60 \\
\hline
\end{tabular}

